\title{
Effect of oxidation on charge localization and transport in a single layer of silicon nanocrystals
}

\author{
R. Krishnan ${ }^{\text {a) }}$ \\ Department of Electrical and Computer Engineering, University of Rochester, Rochester, New York 14627 \\ Q. Xie, J. Kulik, X. D. Wang, and S. Lu \\ Process and Materials Characterization Laboratory, APRDL, SPS, Motorola, Arizona 85284 \\ M. Molinari \\ Department of Electrical and Computer Engineering, University of Rochester, Rochester, New York 14627 \\ Y. Gao \\ Department of Physics and Astronomy, University of Rochester, Rochester, New York 14627 \\ T. D. Krauss \\ Department of Chemistry, University of Rochester, Rochester, New York 14627 \\ P. M. Fauchet \\ Department of Electrical and Computer Engineering, University of Rochester, Rochester, New York 14627
}

(Received 14 January 2004; accepted 29 March 2004)

\begin{abstract}
The effect of oxidation on charge transport and retention within a sheet of silicon ( $\mathrm{Si}$ ) nanocrystals was investigated with an electrostatic force microscope. Single layers of nanocrystals with smooth and abrupt $\mathrm{Si} / \mathrm{SiO}_{2}$ interfaces were prepared by thermal crystallization of thin amorphous Si layers, followed by an oxidation treatment for isolating the nanocrystals. Controlled amounts of charge were injected into the nanocrystals and their in-plane diffusion was monitored in real time. Rapid transport of the injected charge occurred for the nonoxidized nanocrystals. Oxidation of the nanocrystal layer resulted in suppression of lateral transport. The nanocrystals oxidized for $30 \mathrm{~min}$ retained the injected charge in a well-defined, localized region with retention times of the order of several days. These long-term charge retention characteristics indicate that nanocrystals prepared by this process could be attractive candidates for nonvolatile memory applications. (C) 2004 American Institute of Physics. [DOI: 10.1063/1.1751632]
\end{abstract}

\section{INTRODUCTION}

Over the past decade, silicon $(\mathrm{Si})$ nanocrystals have generated tremendous scientific interest due to their potential applications in optical and electronic devices. In memory cells, Si nanocrystals can serve as charge storage nodes and offer several benefits in comparison to continuous polysilicon layers. ${ }^{1}$ Various studies have shown that although the nanocrystal memory cells are extremely promising in terms of low power consumption, scalability, and endurance to write-erase cycles, they have inferior charge retention characteristics in comparison to the 10 year requirement of commercial flash electrically erasable programmable read only memory (EEPROM). ${ }^{1-3}$ To make these devices truly nonvolatile with long charge retention and low refresh times, the nanocrystals must be electrically isolated. Furthermore, they should be located at a fixed depth and have a narrow size distribution in order to avoid fluctuations in the charging voltage. 4

None of the currently used nanocrystal fabrication technologies can fulfill all these criteria. For example, the most commonly adopted methods are implantation of $\mathrm{Si}$ into $\mathrm{SiO}_{2}{ }^{5,6}$ and annealing of $\mathrm{SiO}_{x}(x<2)$ films. ${ }^{7}$ Both of these

\footnotetext{
a) Author to whom correspondence should be addressed; electronic mail: rishi@ece.rochester.edu.
}

techniques produce nanocrystals with a very broad size distribution ( $20 \%$ to $40 \%$ deviation from average size) and poor spatial control. ${ }^{8}$ The ion implantation process also damages the interface between crystalline silicon $(c-\mathrm{Si})$ and amorphous $\mathrm{SiO}_{2}\left(a-\mathrm{SiO}_{2}\right)$ and generates defects in the oxide through which the injected charge leaks and drains the memory cell. ${ }^{9}$ Due to these limitations, nanocrystal memories have not yet replaced conventional EEPROMS and are mostly restricted to applications such as dynamic random access memories where benefits associated with using nanocrystals far outweigh the requirements for having perfect nonvolatility. ${ }^{7}$ If alternative processes or structures with superior charge storage characteristics were developed, they would have a significant impact in the realization of nonvolatile nanocrystal memories.

Tsybeskov et al. ${ }^{10}$ and Grom et al. ${ }^{11}$ reported fabrication of Si nanocrystals sandwiched between nanometer thick layers of $a-\mathrm{SiO}_{2}$ by thermal crystallization of $a-\mathrm{SiO}_{2} /$ amorphous $\mathrm{Si}(a-\mathrm{Si}) / a-\mathrm{SiO}_{2}$ multilayers. This fabrication methodology produces nanocrystals with well controlled sizes in the vertical (growth) direction, is completely compatible with existing microelectronic processes, and allows for precise positioning of the nanocrystal layers. However, a drawback of this technique is that the lateral isolation of the nanocrystals is poor with many of them touching each 
other. ${ }^{12,13}$ In this work, we used a low temperature oxidation treatment for isolating the nanocrystals and studied the resulting electrical isolation with mesoscopic lateral transport measurements. These measurements were performed on single layers of nanocrystals and differ from previous works that have almost exclusively focused on vertical transport. ${ }^{14-16}$ We expect the experiments and results presented here will be useful not only for nonvolatile memories but also for other applications where Si nanocrystals are envisioned to play an important role such as single electron transistors ${ }^{17,18}$ and lasers. ${ }^{19}$

For studying the nanocrystal isolation, controlled amounts of charge were injected within a small circular disk and the subsequent charge diffusion was monitored. The charge was injected in the nanocrystals with an extremely sharp, conductive probe (radius of curvature $=17 \mathrm{~nm}$ ) and imaged with an electrostatic force microscope (EFM). A 30 min oxidation treatment resulted in an effective electrical isolation of the nanocrystals and the injected charge was retained within the originally charged nanocrystals. In the nonoxidized nanocrystals, a significant electrical cross talk caused a rapid diffusion of charges and a pronounced expansion in the charged area. Complementary measurements involving transmission electron microscopy (TEM), annular dark field scanning transmission electron microscopy (ADFSTEM), and x-ray photoelectron spectroscopy (XPS) were performed on these samples to determine the size of the nanocrystals, their separation, and the stoichiometry of $\mathrm{SiO}_{2}$ resulting from the oxidation treatments. We observed that thermal oxidation resulted in oxygen $\left(\mathrm{O}_{2}\right)$ diffusing through the nanocrystal layer, reacting with the grain boundaries and isolating the nanocrystals with $\mathrm{SiO}_{2}$.

\section{EXPERIMENT}

$\mathrm{Si}$ wafers (4 in. diameter, phosphorus doped, and resistivity $\sim 0.05 \Omega \mathrm{cm}$ ) were cleaned using standard cleaning processes. The wafers were immersed in a 100:1 hydrofluoric acid bath for $60 \mathrm{~s}$ to strip off the native oxide and the bare wafers were thermally oxidized in a furnace tube under dry $\mathrm{O}_{2}$ flow to grow $25 \mathrm{~nm}$ thick $a-\mathrm{SiO}_{2}$. The oxidized wafers were introduced into a sputtering tool and a thin $a$-Si layer was grown by radio-frequency magnetron sputtering of an undoped $c$-Si target. Without breaking vacuum, a $2 \mathrm{~nm}$ thick $a-\mathrm{SiO}_{2}$ layer was deposited on top of this $a$-Si layer to form an $\left(a-\mathrm{SiO}_{2} / a-\mathrm{Si} / a-\mathrm{SiO}_{2}\right)$ sandwich structure. Si nanocrystals were formed by thermally treating this sandwich structure with a rapid thermal anneal $\left(950^{\circ} \mathrm{C}, 30 \mathrm{~s}\right)$ followed by a 10 min equilibrium furnace anneal at $1050^{\circ} \mathrm{C}$ in $\mathrm{N}_{2}$ ambient. Separate wafers with nanocrystals were then oxidized in dry $\mathrm{O}_{2}$ at $750^{\circ} \mathrm{C}(1 \mathrm{~atm})$ for 0,15 , and $30 \mathrm{~min}$ and analyzed by atomic force microscopy (AFM), TEM, XPS, and EFM. For ADFSTEM, two period nanocrystal layers sandwiched between $a-\mathrm{SiO}_{2}$ were prepared following identical deposition and annealing conditions and were oxidized in dry $\mathrm{O}_{2}$ at $750{ }^{\circ} \mathrm{C}(1 \mathrm{~atm})$ for 0 and $30 \mathrm{~min}$.

The EFM measurements were performed with a Digital Instruments Nanoscope IIIa Bioscope AFM. Commercially available metal coated $n^{+} \mathrm{Si}$ cantilevers with a resonant fre-

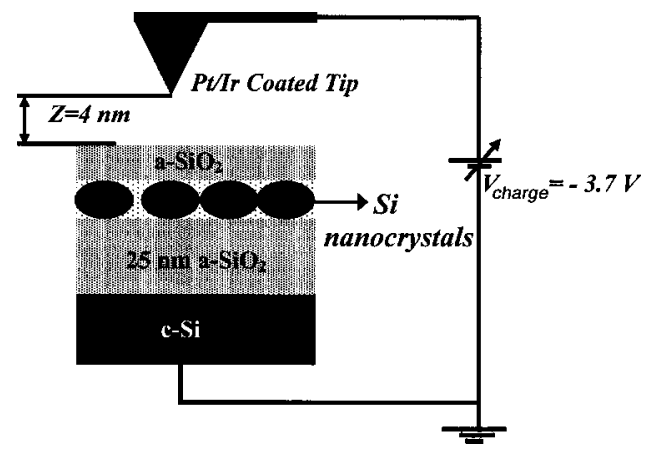

FIG. 1. Electrical schematic of the EFM setup during charge injection.

quency of $\sim 65 \mathrm{KHz}$ and spring constant $\sim 2.0 \mathrm{~N} / \mathrm{m}$ were used. The nanocrystal samples were heated at $450{ }^{\circ} \mathrm{C}$ in high vacuum to remove any moisture adsorbed within the films and immediately introduced into a $\mathrm{N}_{2}$ purged EFM enclosure. All subsequent experiments involving charging of nanocrystals and imaging of the stored charges were conducted within this enclosure. For charging the nanocrystals, the following protocol was adopted: After disabling the slow and fast scan axes, the oscillating cantilever (free rms amplitude $=0.5 \mathrm{~V}$ ) was lowered until the tip was $4 \mathrm{~nm}$ above the sample surface and a negative bias $\left(V_{\text {charge }}=-3.7 \mathrm{~V}\right)$ was then applied to it for $30 \mathrm{~s}$ with the sample stage grounded (Fig. 1).

After charging the nanocrystals, EFM images were acquired in a two-pass procedure. ${ }^{20,21}$ In the first pass, a line scan of the sample topography was obtained during normal tapping mode imaging with no bias applied to the tip. In the second pass, the tip was lifted $20 \mathrm{~nm}$ above the sample surface, an ac bias $\left(V_{\mathrm{ac}}=\mathrm{V}_{0} \sin \omega \mathrm{t} ; V_{0} \sim 0.2 \mathrm{~V}, \omega=400 \mathrm{~Hz}\right)$ superimposed on a dc bias $\left(V_{\mathrm{dc}} \sim-0.7 \mathrm{~V} \pm 0.01 \mathrm{~V}\right)$ applied to it and the lifted tip rescanned above the line traced in the first pass. The magnitude of the dc bias was chosen so as to null out the contact potential difference between the tip and sample (Appendix). As the tip moves along the nanocrystals at a fixed height, it responds to the presence of electrostatic forces created by the charge on the nanocrystals, its image and charge induced on the tip by the ac bias. The Coulomb force resulting from these charges causes a resonant frequency shift in the cantilever oscillation that modulates at $\omega$. These shifts, $\Delta \nu(\omega)$ were recorded with a lock in amplifier and used for quantifying the charge in the nanocrystals.

For each set of images, the $\Delta \nu(\omega)$ values were obtained by taking a line section through the center of the charged region. The temporal evolution of the $\Delta \nu(\omega)$ signal was monitored by continuously acquiring EFM images over a surface area encircling the charged region. The average time to acquire a complete image was 6 min $(256 \times 256$ pixels, scan rate $=1.3 \mathrm{~Hz}$ ). Each sample was investigated with a new tip, but for a given sample, the same tip was used for both charging and imaging. The integrity of the tip was confirmed at the end of the experiments with independent scanning electron microscopy images or by imaging nanoparticles with known sizes and checking for convolution effects or imaging artifacts. 
(a)

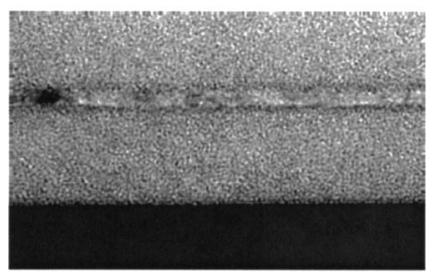

$\underline{20 \mathrm{~nm}}$

(b)

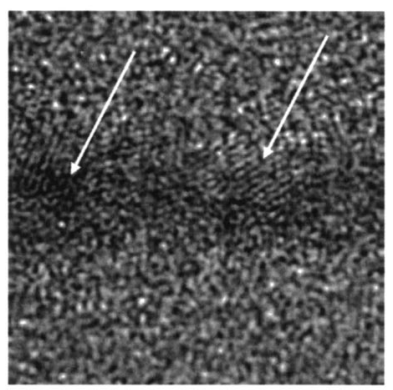

$5 \mathrm{~nm}$

FIG. 2. TEM images for a nonoxidized nanocrystal layer (a) bright field image and (b) high resolution image. Lattice fringes from the nanocrystals are indicated by arrows.

Cross-sectional TEM samples were prepared by conventional tripod polishing, followed by Ar ion milling to thin down the samples to electron transparency. Bright field and high resolution lattice imaging were performed with a Philips CM200 microscope equipped with a $200 \mathrm{KeV}$ field emitting gun. The ADFSTEM measurements were performed with a $0.6 \mathrm{~nm}$ diameter electron probe.

The XPS measurements were obtained with a Surface Science Instrument SSX-100 equipped with a monochromatic Al K $\alpha$ source $(1486.6 \mathrm{eV})$. The spectra were collected with a resolution of $0.7 \mathrm{eV}$ and the base pressure in the XPS chamber during the analysis was $\sim 10^{-10}$ Torr. The energy scale in the measurements was calibrated using the binding energy of the $\mathrm{C} 1 \mathrm{~S}$ peak at $284.5 \mathrm{eV}$. The spectra are reported after correcting for an inelastic Shirley-type background.

\section{RESULTS AND DISCUSSION}

Annealing the $\left(a-\mathrm{SiO}_{2} / a-\mathrm{Si} / a-\mathrm{SiO}_{2}\right)$ sandwich structure converted the thin $a$-Si layer into a layer composed of nanocrystalline $\mathrm{Si}$ grains. The presence of Si nanocrystals was confirmed by bright field cross-sectional TEM images and high-resolution lattice images (Fig. 2). All the nanocrystals had similar vertical (growth direction) sizes since their growth was constrained on either end by the $a-\mathrm{SiO}_{2}$ layers. The surface morphology of the oxide capped nanocrystal surface was measured by AFM before and after the oxidation treatments and observed to be extremely flat with an average root mean square (rms) roughness of $0.2 \mathrm{~nm}$. Oxidation did not lead to any noticeable increase in the average rms surface roughness but led to a reduction in the nanocrystal layer thickness (Fig. 3). The reduction in nanocrystal layer thickness as a function of the oxidation time was measured by (a)

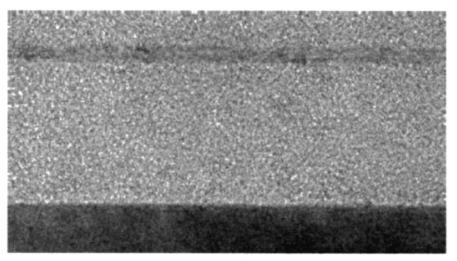

$20 \mathrm{~nm}$

(b)

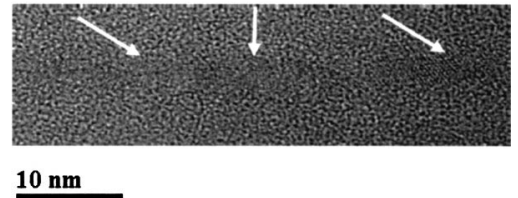

FIG. 3. TEM images of a 30 min oxidized nanocrystal layer showing a thinner nanocrystal layer (a) bright field image and (b) high resolution image. Lattice fringes from the nanocrystals are indicated by arrows.

TEM and is plotted in Fig. 4. In the nonoxidized layer, the nanocrystal layer thickness in the vertical (growth) direction was $5.4 \mathrm{~nm} \pm 0.2 \mathrm{~nm}$ while in the $30 \mathrm{~min}$ oxidized layer, the thickness was reduced to $3 \mathrm{~nm} \pm 0.3 \mathrm{~nm}$. For evaluating the separation in the nanocrystal layer, annular dark field scanning transmission electron microscopy (ADFSTEM) was used. The image contrast in ADFSTEM is independent of orientation of the nanocrystals and originates due to differences in atomic number between $\mathrm{Si}$ (14) and $\mathrm{SiO}_{2}$ (average of 10). In the images shown in Fig. 5, Si appears with a bright contrast whereas $\mathrm{SiO}_{2}$ appears with a dark contrast. In both images, two distinct Si nanocrystal layers can be observed (shown by arrows). In comparison to the nonoxidized nanocrystal layers, significant contrast variations exist within the 30 min oxidized nanocrystal layers. In the latter, the nanocrystal layers appear more discontinuous, indicating increased separation of the nanocrystals. Since this discontinuity is also observed in the bottom nanocrystal layer, it implies that oxygen diffused through the top nanocrystal layer. Oxygen reacts with $\mathrm{Si}$ at the lateral boundaries of the nanocrystals, causing the formation of $\mathrm{SiO}_{2}$ and thus separating the nanocrystals. For the nonoxidized nanocrystal layer, the

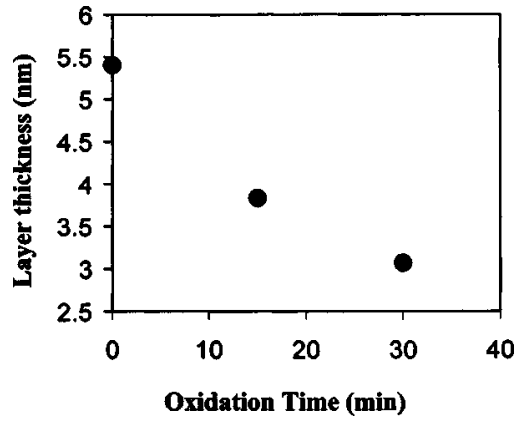

FIG. 4. Thickness of Si nanocrystal layer as a function of oxidation time in dry $\mathrm{O}_{2}$ at $750{ }^{\circ} \mathrm{C}(1 \mathrm{~atm}$.). 
(a)

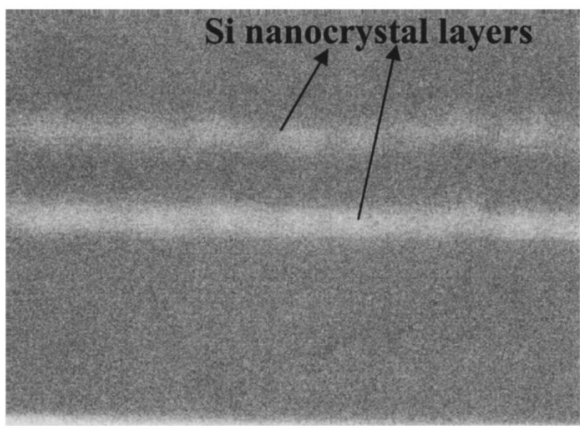

$20 \mathrm{~nm}$

(b)

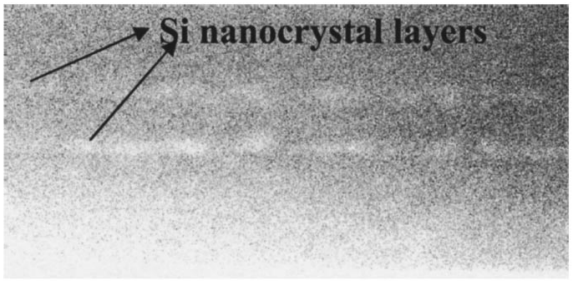

$20 \mathrm{~nm}$

FIG. 5. ADFSTEM images of a two period nanocrystal layer (a) nonoxidized and (b) oxidized at $750{ }^{\circ} \mathrm{C}$ for $30 \mathrm{~min}$. The nanocrystal layers appear with a bright contrast.

spacing between neighboring nanocrystals is less than $1 \mathrm{~nm}$, whereas for the 30 min oxidized nanocrystal layers, it is as large as $3.6 \mathrm{~nm}$.

Figure 6 shows XPS spectra obtained from a $c-\mathrm{Si} / a-\mathrm{SiO}_{2}(25 \mathrm{~nm}) / a-\mathrm{Si}(5.4 \mathrm{~nm}) / a-\mathrm{SiO}_{2}(2 \mathrm{~nm}) \quad$ sandwich structure oxidized for different durations at $750{ }^{\circ} \mathrm{C}$. Each spectrum in the figure consists of two characteristic peaks. The peak located at $103.8 \mathrm{eV}$ is the signature peak for $\mathrm{Si}$ bonded to four $\mathrm{O}$ atoms $\left(\mathrm{SiO}_{2}\right)$ whereas the peak located at $99.3 \mathrm{eV}$ corresponds to a $\mathrm{Si}$ atom tetrahedrally bonded to four $\mathrm{Si}$ atoms. ${ }^{22}$ Because the nanocrystal layer is separated from the substrate by $25 \mathrm{~nm}$ thick $\mathrm{SiO}_{2}$, the entire signal at $99.3 \mathrm{eV}$ originates from $\mathrm{Si}$ in the nanocrystals and the contribution from the $c$-Si substrate is negligible. With increasing oxidation duration, the peak at $99.3 \mathrm{eV}$ reduces and the

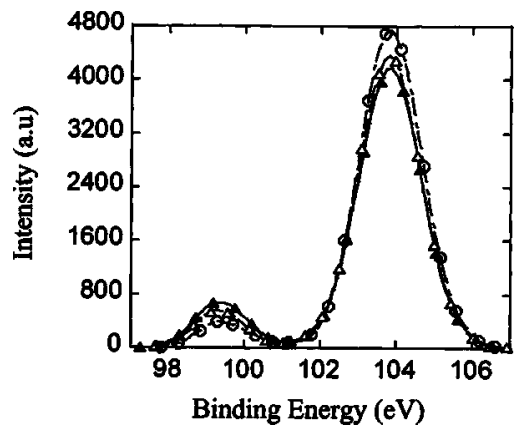

FIG. 6. XPS spectra obtained from a single layer of Si nanocrystal layer capped with $2 \mathrm{~nm}$ of $\mathrm{SiO}_{2}$ and oxidized for different durations. Closed triangle: Nonoxidized nanocrystal layer; open triangle: 15 min oxidized nanocrystal layer; open circle: 30 min oxidized nanocrystal layer.
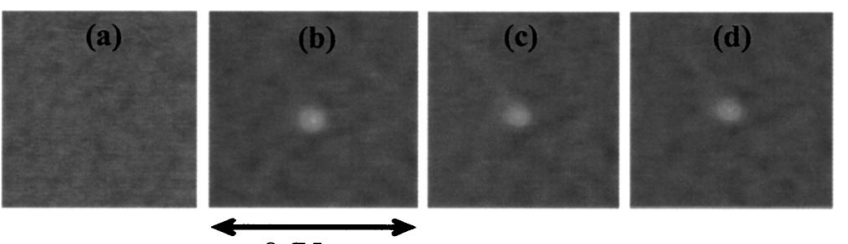

$0.75 \mu \mathrm{m}$
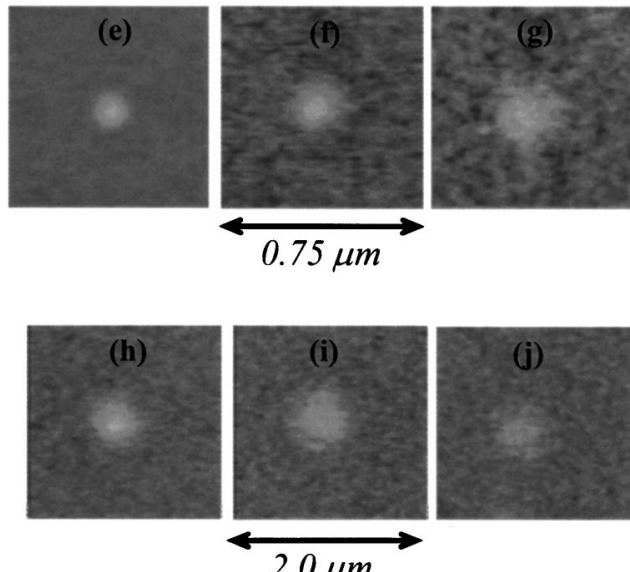

$2.0 \mu m$

FIG. 7. (a) - (d) $\Delta \nu(\omega)$ images acquired during the second pass of the EFM measurements for the $30 \mathrm{~min}$ oxidized nanocrystal layer. The images shown in the panels were obtained (a) before charging and (b) $0.2 \mathrm{~h}(12 \mathrm{~min})$, (c) 3 $\mathrm{h}(180 \mathrm{~min})$, and (d) $24 \mathrm{~h}$ (1440 min) after charging. The full scale of the four images is $0.75 \mu \mathrm{m} \times 0.75 \mu \mathrm{m}$. (e) $-(\mathrm{g}) \Delta \nu(\omega)$ images acquired during the second pass of the EFM measurements for the 15 min oxidized nanocrystal layer. The images shown in the panels were obtained (e) $0.2 \mathrm{~h}$ (12 $\mathrm{min}$ ), (f) $4.2 \mathrm{~h}$ (252 $\mathrm{min}$ ), and (g) $18.1 \mathrm{~h}$ (1086 min) after charging. The full scale of the three images is $0.75 \mu \mathrm{m} \times 0.75 \mu \mathrm{m}$. (h) $-(\mathrm{j}) \Delta \nu(\omega)$ images acquired during the second pass of the EFM measurements for the nonoxidized nanocrystal layer. The images shown in the panels were obtained (h) $0.2 \mathrm{~h}$ (12 min), (i) $0.3 \mathrm{~h}$ (18 min), and (j) $0.8 \mathrm{~h}$ (48 min) after charging. The full scale of the three images is $2.0 \mu \mathrm{m} \times 2.0 \mu \mathrm{m}$.

peak at $103.8 \mathrm{eV}$ increases indicating consumption of $\mathrm{Si}$ in the nanocrystal layer and formation of $\mathrm{SiO}_{2}$. The $\mathrm{SiO}_{2}$ formed during the oxidation process is stoichiometric since no shoulders or peaks (in the region between 100 and 103 $\mathrm{eV}$ ) attributable to the presence of $\mathrm{SiO}_{x}(x<2)$ suboxides are observed. $^{22}$

Figure 7(a) shows the $\Delta \nu(\omega)$ image recorded prior to charging the $30 \mathrm{~min}$ oxidized nanocrystal layer. The $\Delta \nu(\omega)$ image obtained $12 \mathrm{~min}$ after charging the nanocrystals is shown in Fig. 7(b) for comparison. The injected charge appears as a bright contrast [positive $\Delta \nu(\omega)]$ in the $\Delta \nu(\omega)$ image. As per the experimental sign conventions, a positive (negative) $\Delta \nu(\omega)$ signal corresponds to the presence of negative (positive) charges, which implies that the negative tip bias resulted in the injection of electrons into the nanocrystals. The temporal evolution of the EFM images can be observed in Figs. 7(c) and 7(d). The intensity of $\Delta \nu(\omega) / \nu_{0}$ drops slightly from $7.58 \times 10^{-4}$ to $6.6 \times 10^{-4}, 3.0 \mathrm{~h}$ after charge injection and to $5.04 \times 10^{-4}$ after $24.0 \mathrm{~h}$. The full width half maximum (FWHM) remained virtually unchanged at $62 \mathrm{~nm} \pm 3 \mathrm{~nm}$. The stability of the EFM images over this duration with small reduction in $\Delta \nu(\omega)$ signal strength shows that the injected electrons remained localized with very slow detrapping kinetics. 
(a)

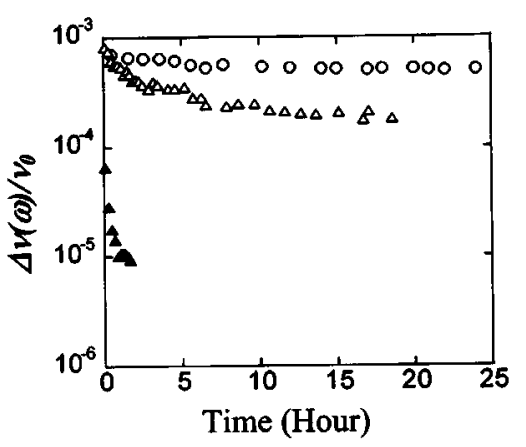

(b)

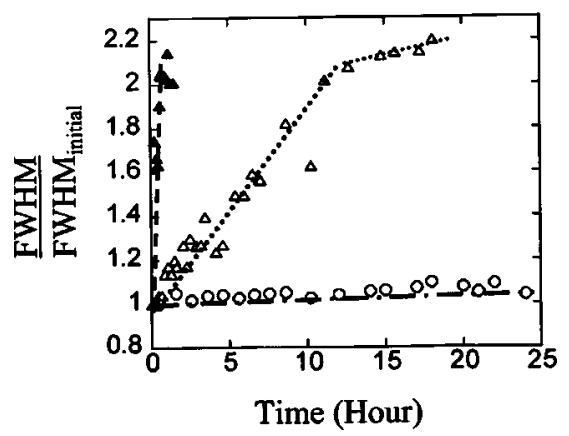

FIG. 8. (a) Time decay of the $\Delta \nu(\omega)$ signal after charge injection. For the 30 min oxidized nanocrystal sample, the decay is nearly exponential. (b) FWHM evolution of the $\Delta \nu(\omega)$ curves. Open circles: 30 min oxidized nancrystal layer; open triangles: 15 min oxidized nanocrystal layer; closed triangles: nonoxidized nanocrystal layer. The lines are only a guide for the eye.

In comparison, the $\Delta \nu(\omega)$ images obtained for the nanocrystal layer oxidized for 15 min showed a faster reduction in the signal contrast after charging [Figs. $7(\mathrm{e})-7(\mathrm{~g})]$. In this case, the $\Delta \nu(\omega) / \nu_{0}$ signal strength decreased from 8.40 $\times 10^{-4}$ to $1.77 \times 10^{-4}$ in $18.1 \mathrm{~h}(1086 \mathrm{~min})$ after charge injection and was accompanied by a simultaneous increase in the FWHM of the $\Delta \nu(\omega)$ profile from 91 to $200 \mathrm{~nm}$. The $\Delta \nu(\omega)$ images show a clear spatial expansion, which implies that over time the electrons were transported laterally within the nanocrystal layer. A similar measurement on the nonoxidized nanocrystal layer showed that it was very leaky and the electrons were rapidly removed from the charged nanocrystals. This results in an expansion of the charged area and reduction in $\Delta \nu(\omega)$ signal strength after charging the nanocrystals [Figs. 7(h) $-7(\mathrm{j})$ ]. The FWHM increased from 287 to $585 \mathrm{~nm}$ in $0.8 \mathrm{~h}(48 \mathrm{~min})$ after charging during which the $\Delta \nu(\omega) / \nu_{0}$ strength decreased from $4.86 \times 10^{-5}$ to 8.88 $\times 10^{-6}$.

To characterize the samples, we define a time constant $\tau$ as the time over which the $\Delta \nu(\omega)$ signal drops to 1/e of its maximum value. From the discharge transients shown in Fig. 8 (a), it can be noted that $\tau=5.66 \mathrm{~h}$ for the $15 \mathrm{~min}$ oxidized sample, while for the nonoxidized sample $\tau=0.53 \mathrm{~h}$. For the 30 min oxidized nanocrystal sample, the $\Delta \nu(\omega)$ signal decayed to only $60 \%$ of its original value within the measurement span of $24 \mathrm{~h}$. The 1/e point is determined by curve fitting the $\Delta \nu(\omega) / \nu_{0}$ decay curve and the time constant cal-

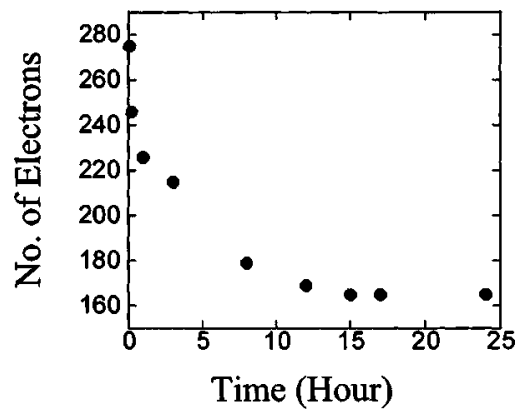

FIG. 9. Charge decay over time. The charges were calculated from the measured $\Delta \nu(\omega)$ signals by utilizing the model outlined in the Appendix.

culated this way is $\sim 371 \mathrm{~h}(\sim 15.45$ days $)$. The continuous evolution of the $\Delta \nu(\omega)$ FWHM during decay is shown in Fig. 8(b). For both the nonoxidized and 15 min oxidized sample, the FWHM of the $\Delta \nu(\omega)$ profile continuously increased with time, indicating significant lateral transport within the nanocrystal layer. On the other hand, for the $30 \mathrm{~min}$ oxidized sample the $\Delta \nu(\omega)$ FWHM remained fixed during the entire duration of the measurement.

In all three samples that were studied by EFM, the nanocrystals were isolated from the $c$-Si substrate by a 25 $\mathrm{nm}$ thick thermally grown $\mathrm{SiO}_{2}$. Direct vertical transport of charge into the substrate is thus minimal. Lateral movement of charge along the nanocrystal plane occurs by transport of electrons from one nanocrystal to another. For a transport process dependent on tunneling of electrons from a charged to an uncharged nanocrystal, the dependence of charge retention time $\tau$ on nanocrystal separation $(d)$ is given by ${ }^{23}$

$$
\tau \propto \exp \left[2 d\left(2 m q \varphi / \hbar^{2}\right)^{1 / 2}\right] .
$$

Here, $m$ is the effective mass of electrons and $\varphi$ is the potential barrier height. This equation predicts that $\tau$ should increase with an increase in nanocrystal separation. Our observation is in qualitative agreement with this expectation. The lateral charge transport is fastest for the nonoxidized nanocrystal layer containing closely spaced nanocrystals ( $d$ $<1 \mathrm{~nm}$ ) and is slowest for the 30 min oxidized nanocrystal layer containing separated nanocrystals $(d \leqslant 3.6 \mathrm{~nm})$.

Using a model outlined in the Appendix, the measured $\Delta \nu(\omega)$ magnitudes are utilized to quantify the amount of charge. For the 30 min oxidized nanocrystal sample, a total of 275 electrons is calculated at time $t=6 \mathrm{~min}$. The charge magnitude at subsequent intervals is shown in Fig. 9. After 3 $\mathrm{h}$, the charge magnitude has dropped to 215 electrons and after $24 \mathrm{~h}$ to 165 electrons. Thus, only a $40 \%$ reduction in the amount of charge occurs in $24 \mathrm{~h}$. This decay is significantly slower than that reported so far in localized charge retention experiments in $\mathrm{Si}$ nanocrystals. ${ }^{9,24-27}$ For example, Boer et al. $^{24}$ and Guillemot et al. ${ }^{25}$ have performed EFM measurements on Si nanocrystals prepared by aerosol synthesis and thermal annealing of nonstoichiometric $\mathrm{SiO}_{x}$, respectively. In both their samples the nanocrystals were isolated from the substrate by thick $\mathrm{SiO}_{2}$ (thickness $\geqslant 25 \mathrm{~nm}$ ), yet the injected charges decayed quickly with retention times lower than $4 \mathrm{~h}$. In similar EFM measurements on Si nanocrystals prepared 
by ion-implantation, ${ }^{9}$ a rapid decay at a rate of $35 \pm 15$ electrons/min occurred for an injected charge of $350 \pm 90$ electrons.

The charge decay curve in Fig. 9 also indicates that there is a permanent loss of electrons from the nanocrystals over time. Experiments with different tip-sample contact times and lift heights suggest that direct transfer of electrons from the nanocrystals into the EFM tip is not a significant loss path. We suggest two possibilities that could explain the apparent loss of electrons: (1) A small amount of vertical transport due to the presence of defects in the oxide and (2) recombination of electrons with residual positive carriers in the sample. These mechanisms should be clarified with further experiments involving depth analysis of oxide defects and ionic impurities.

From an extrapolation of the decay curve, we estimated that for the $30 \mathrm{~min}$ oxidized sample $\Delta \nu(\omega) / \nu_{0}$ would reduce to $1 / e$ of its initial value after $371 \mathrm{~h}$ (15.45 days). This corresponds to 101 electrons still being present in the originally charged region. The long term localized charge retention characteristics in the oxidized nanocrystals can be directly attributed to their better encapsulation with $\mathrm{SiO}_{2}$ and increased spatial isolation. The long charge retention time coupled with the benefits of smooth surface morphology, monodisperse nanocrystal size distribution, low density of $\mathrm{Si} / \mathrm{a}-\mathrm{SiO}_{2}$ interface defects, and low $\mathrm{Si} / \mathrm{a}-\mathrm{SiO}_{2}$ interface roughness ${ }^{11}$ makes Si nanocrystals prepared by thermal crystallization potential candidates for nonvolatile memory devices.

\section{CONCLUSION}

This work demonstrates fabrication of single nanocrystal sheets containing electrically isolated nanocrystals that can be placed at fixed depths. The high quality of isolation is achieved by oxidizing nanocrystal grain boundaries and effectively encapsulating the nanocrystal surface with $\mathrm{SiO}_{2}$. The possibility of making these nanocrystals by a simple process completely compatible with conventional microelectronic fabrication technology provides attractive options for nanocrystal memories.

\section{ACKNOWLEDGMENTS}

This work was made possible by financial support from the Army Research Office (ARO) with additional support from the Semiconductor Research Corporation (SRC). We thank S. Zorba for help with XPS measurements and G. Tam for preparing the samples for the STEM measurements.

\section{APPENDIX: CHARGE QUANTIFICATION}

For a simple harmonic oscillator, the relative shift in the cantilever resonant frequency $\Delta \nu(\omega) / \nu_{0}$ is related to the spring constant $(k)$ and the force gradient $(d F / d Z)$ in the vertical $(Z)$ direction by ${ }^{28}$

$$
\frac{\Delta \nu(\omega)}{\nu_{0}}=-\frac{1}{2 k} \times \frac{d F(\omega)}{d Z} .
$$

The total force acting on the tip can be expressed as ${ }^{20,21,29}$

$$
\begin{aligned}
F(\omega)= & \left\{\left(V_{\mathrm{dc}}+\phi\right) V_{\mathrm{ac}} \frac{d C(z)}{d Z}\right. \\
& \left.+E_{z}\left[C(z) V_{\mathrm{ac}}+\sum_{i} \sum_{j} q_{i, j}^{\mathrm{tip}}\right]\right\},
\end{aligned}
$$

where $\phi$ is the contact potential difference between the tip and the sample, $C$ is the tipsample capacitance, $E_{z}$ is the total electric field acting in the $Z$ direction, and $q_{i, j}^{\text {tip }}$ is the charge induced on the tip due to a charge located at point $(i, j)$ on the nanocrystal surface.

The first term in Eq. (A2) was nulled out during the experiments by setting $V_{\mathrm{dc}}=-\varphi$ (in our case $V_{\mathrm{dc}}=-0.7 \mathrm{~V}$ $\pm 0.01 \mathrm{~V}) .^{20,21}$ For estimating the remaining force terms, knowledge of the tip-sample capacitance is required. We follow the approach cited in Ref. 29 and model the EFM probe as a cone with a sphere on one end and a parallel plate on the other end. To estimate the electric field and induced charges, the charge distribution on the nanocrystal surface was taken to be a two-dimensional gaussian profile $(\mathrm{FWHM}=62 \mathrm{~nm})$ imposed on a grid with $5 \mathrm{~nm} \times 5 \mathrm{~nm}$ square pixels. The charge grid was placed at the center of the nanocrystal layer and the method of images ${ }^{30}$ was then used to calculate the charge induced on the tip $q_{i, j}^{\text {tip }}$ and its location $Z^{\text {tip }}$.

$$
\begin{aligned}
& q_{i, j}^{\mathrm{tip}}=-q_{i, j} \times \frac{R}{R+Z}, \\
& Z^{\mathrm{tip}}=Z+R-\frac{R^{2}}{(R+Z)} .
\end{aligned}
$$

In these equations, $R$ is the radius of curvature of the spherical part of the tip. The image charge induced on the $c-\mathrm{Si}$ substrate was also calculated and its influence on the total force was found to be negligible due to the $25 \mathrm{~nm}$ thick $\mathrm{SiO}_{2}$ layer separating it from the charges in the nanocrystal layer.

${ }^{1}$ S. Tiwari, F. Rana, H. Hanafi, A. Hartstein, E. F. Crabbe, and K. Chan, Appl. Phys. Lett. 68, 1377 (1996).

${ }^{2}$ G. Iannaccone and P. Coli, Appl. Phys. Lett. 78, 2046 (2001).

${ }^{3}$ M. L. Ostraat, J. W. De Blauwe, M. L. Green, L. D. Bell, M. L. Brongersma, J. Casperson, R. C. Flagan, and H. A. Atwater, Appl. Phys. Lett. 79, 433 (2001).

${ }^{4}$ Y. C. King, T. J. King, and C. Hu, IEEE Trans. Electron Devices 48, 696 (2001).

${ }^{5}$ H. I. Hanafi, S. Tiwari, and I. Khan, IEEE Trans. Electron Devices ED-43, 1553 (1996).

${ }^{6}$ E. Kapetanakis, P. Normand, D. Tsoukalas, K. Beltsios, J. Stoemenos, S. Zhang, and J. Van den Berg, Appl. Phys. Lett. 77, 3450 (2000).

${ }^{7}$ Y. C. King, T. J. King, and C. Hu, IEEE Electron Device Lett. 20, 409 (1999).

${ }^{8}$ B. Garrido Fernandez, M. López, C. Garcia, A. Pérez-Rodriguéz, J. R. Morante, C. Bonafos, M. Carrada, and A. Claverie, J. Appl. Phys. 91, 798 (2002)

${ }^{9}$ E. Boer, M. L. Brongersma, H. A. Atwater, R. C. Flagan, and L. D. Bell, Appl. Phys. Lett. 79, 791 (2001).

${ }^{10}$ L. Tsybeskov, K. D. Hirschman, S. P. Duttagupta, M. Zacharias, P. M. Fauchet, J. P. McCaffrey, and D. J. Lockwood, Appl. Phys. Lett. 72, 43 (1998).

${ }^{11}$ G. F. Grom, D. J. Lockwood, J. P. McCaffrey, H. J. Labbe, P. M. Fauchet, B. White, J. Diener, D. Kovalev, F. Koch, and L. Tsybeskov, Nature (London) 407, 358 (2000).

${ }^{12}$ M. Zacharias, J. Blasing, P. Veit, L. Tsybeskov, K. Hirschman, and P. M. Fauchet, Appl. Phys. Lett. 74, 2614 (1999).

${ }^{13}$ M. Zacharias, L. Tsybeskov, K. D. Hirschman, P. M. Fauchet, J. Blasing, P. Kohlert, and P. J. Veit, J. Non-Cryst. Solids 227-230, 1132 (1998). 
${ }^{14}$ L. Tsybeskov, G. F. Grom, R. Krishnan, L. Montes, P. M. Fauchet, D. Kovalev, J. Diener, V. Timoshenko, F. Koch, J. P. McCaffrey, J. M. Baribeau, G. I. Sproule, D. J. Lockwood, Y. M. Niquet, C. Delerue, and G. Allan, Europhys. Lett. 55, 552 (2001).

${ }^{15}$ L. Tsybeskov, G. F. Grom, P. M. Fauchet, J. P. McCaffrey, J. M. Baribeau, G. I. Sproule, and D. J. Lockwood, Appl. Phys. Lett. 75, 2265 (1999).

${ }^{16}$ D. N. Kouvatos, V. Ioannou-Sougleridis, and A. G. Nassiopoulou, Mater. Sci. Eng., B 101, 270 (2003).

${ }^{17}$ Y. Furuta, H. Mizuta, K. Nakazato, T. Kamiya, Y. T. Tan, Z. A. K. Durrani, and K. Taniguchi, Jpn. J. Appl. Phys., Part 1 41, 2675 (2002).

${ }^{18}$ T. W. Kim, D. C. Choo, J. H. Shim, and S. O. Kang, Appl. Phys. Lett. 80, 2168 (2002).

${ }^{19}$ J. Ruan, P. M. Fauchet, L. Dal Negro, M. Cazzaneli, and L. Pavesi, Appl. Phys. Lett. 83, 5479 (2003).

${ }^{20}$ T. D. Krauss and L. E. Brus, Phys. Rev. Lett. 83, 4840 (1999).

${ }^{21}$ T. D. Krauss, S. O'Brien, and L. E. Brus, J. Phys. Chem. B 105, 1725 (2001).
${ }^{22}$ F. Iacona, S. Lombardo, and S. U. Campisano, J. Vac. Sci. Technol. B 14, 2693 (1996)

${ }^{23}$ D. M. Schaadt, E. T. Yu, S. Sankar, and A. E. Berkowitz, J. Electron. Mater. 29, 1299 (2000).

${ }^{24}$ E. A. Boer, L. D. Bell, M. L. Brongersma, H. A. Atwater, M. L. Ostraat, and R. C. Flagan, Appl. Phys. Lett. 78, 3133 (2001).

${ }^{25}$ C. Guillemot, P. Budau, J. Chevrier, F. Marchi, F. Comin, C. Alandi, F. Bertin, N. Buffet, C. Wyon, and P. Mur, Europhys. Lett. 59, 566 (2002).

${ }^{26}$ S. Banerjee, M. A. Salem, and S. Oda, Appl. Phys. Lett. 83, 3788 (2003).

${ }^{27}$ S. Decossas, F. Mazen, T. Baron, A. Souifi, and G. Brémond, Physica E (Amsterdam) 17, 543 (2003).

${ }^{28}$ Y. Martins, C. C. Williams, and H. K. Wickramasinghe, J. Appl. Phys. 61, 4723 (1987).

${ }^{29}$ O. Cherniavskaya, L. Chen, W. Weng, L. Yuditsky, and L. E. Brus, J. Phys. Chem. 107, 1525 (2003).

${ }^{30}$ J. D. Jackson, Classical Electrodynamics, 2nd ed. (Wiley, New York, 1974), Chap. 2. 